\title{
Enhancement of the AISI 5140 Cold Heading Wire Steel Spheroidization by Adequate Control of the Initial As-Rolled Microstructure
}

\author{
Jon Arruabarrena ${ }^{1,2, *}$ and Jose M. Rodriguez-Ibabe ${ }^{1,2}$ (D) \\ 1 CEIT-Basque Research and Technology Alliance (BRTA), Manuel Lardizabal 15, 20018 Donostia-San Sebastián, \\ Spain; jmribabe@ceit.es \\ 2 Mechanical and Materials Engineering Department, Universidad de Navarra-Tecnun, Manuel Lardizabal 13, \\ 20018 Donostia-San Sebastián, Spain \\ * Correspondence: jonarruabarrena@ceit.es
}

check for

updates

Citation: Arruabarrena, J.; Rodriguez-Ibabe, J.M. Enhancement of the AISI 5140 Cold Heading Wire Steel Spheroidization by Adequate

Control of the Initial As-Rolled

Microstructure. Metals 2021, 11, 219. https://doi.org/10.3390/met11020219

Received: 5 January 2021

Accepted: 24 January 2021

Published: 27 January 2021

Publisher's Note: MDPI stays neutral with regard to jurisdictional claims in published maps and institutional affiliations.

Copyright: (c) 2021 by the authors. Licensee MDPI, Basel, Switzerland. This article is an open access article distributed under the terms and conditions of the Creative Commons Attribution (CC BY) license (https:// creativecommons.org/licenses/by/ $4.0 /)$.

\begin{abstract}
The effect of the initial microstructure and soft annealing temperature on cementite spheroidization and microstructure softening is studied on an AISI 5140 hot-rolled wire. In coarse pearlite microstructure $(\lambda: 0.27 \mu \mathrm{m})$, the cementite spheroidization progresses slowly under subcritical treatment, and the microstructure does not achieve the minimum G2/L2 IFI rating defined in the ASTM F2282 to be used in cold forming operations under any of the annealing treatment studies. Fine pearlite $(\lambda: 0.10 \mu \mathrm{m})$ and upper bainite microstructures are more prone to spheroidization, and the minimum G2/L2 IFI rating is achieved under subcritical annealing at $720{ }^{\circ} \mathrm{C}$ for $6 \mathrm{~h}$. Independent of the initial microstructure, even in the case of martensite, low hardness values within 165-195 HV are attained after imposing a $10 \mathrm{~h}$ long treatment at $720^{\circ} \mathrm{C}$. Annealing treatments conducted at $660^{\circ} \mathrm{C}$ and $600^{\circ} \mathrm{C}$ on pearlitic microstructures give rise to very poor softening. The G2/L2 rating is not achieved in any of the treatments applied at these two temperatures in this study. In pearlitic microstructures, the spheroidization progresses according to a fault migration mechanism, enhanced by the presence of defects such as lamella terminations, holes, and kinks. In the upper bainite, the row-like disposition of the cementite along the ferrite lath interface provides necks where dissolution and consequent lamellae break-up take place quickly under annealing.
\end{abstract}

Keywords: cementite; cold forming; spheroidization; annealing; AISI 5140

\section{Introduction}

Medium carbon steel wires are widely employed for the manufacturing of mechanical fasteners by cold heading operations. For such procedures, the wire must exhibit good workability properties in order to avoid the occurrence of defects, such as folds and shear cracks at the headed parts due to the high loads involved in the process [1]. It also requires a low hardness to enhance tool life. The microstructure, and hence the mechanical properties of the wire, is highly dependent on the conditions in which the cooling stage is carried out in the last step of the manufacturing process after hot rolling. The cooling process initiates after the ring formation in a laying head and continues along the cooling conveyor, where $\gamma \rightarrow \alpha$ phase transformation takes place [2-4]. The cooling conveyor offers a wide range of cooling possibilities that can produce coarse pearlitic to bainitic microstructures and a mixture of both depending on the conveyor speed or the air flow pumped onto the loops [5,6], the diameter of the wire, and the composition of the steel. Commonly, the cooling strategy after hot rolling is devoted to providing soft microstructures that allow the wires to be worked in their as-rolled condition [7-9]. When the cold forming operation involves many blows and/or strains above 1.6, an enhanced workability that cannot be reached in the as-hot rolled condition is demanded, so an intermediate spheroidization softening treatment prior to cold forming needs to be applied [10-13]. According to the 
ASTM F2282, the spheroidized can be categorized in different ratings by the comparison of micrographs at $\times 1000$ magnification with different patterns shown in the standard. The rating varies from G0, which corresponds to a fully spheroidized microstructure with a 100\% spheroidization frequency, to G5/L5 for microstructures consisting of pearlite and ferrite that show no spheroidization characteristics. The rating in between these two exhibits partial cementite spheroidization. The standard includes the letters $\mathrm{G}$ or $\mathrm{L}$ before the number as an indication of the globular or lamellar nature of the microstructure, respectively. For the production of fasteners, wires must exhibit a minimum test rating of G2/L2 as quality assurance for proper workability [10]. That corresponds to a condition where most of the cementites exhibit spheroidal shapes with an average distribution, and few lamellar cementites might be present The standard also requires that the microstructure must fulfil a spheroidization frequency higher than $60 \%$.

The spheroidization treatment is usually performed by subcritical treatments that consist of maintaining the stock at a temperature below the critical temperature A1 [14-18]. Spheroidization progresses faster when the cementites are in the form of discrete particles, as in bainite or tempered martensite, rather than in lamellar morphology [19-23], so the initial cementite features in the as-rolled rod play a crucial role in the kinetics involving spheroidization. Conventionally, high energy and time consumption are involved, and consequently this constitutes the most expensive stage of the production [7]. The holding times at subcritical temperatures vary notably depending on the alloy content and the required degree of coarsening, varying from $10 \mathrm{up}$ to $24 \mathrm{~h}$ [24].

Several models have been proposed for the spheroidization mechanism of pearlite; nevertheless, the main mechanism for the spheroidization under conventional annealing still remains unclear. Some reports have adopted Mullins' modified perturbation model [25] to explain the instability of the lamellar structures, while others found that the initiation and development of the break-up of cementite lamellae were associated with lamellar faults [26-30]. The cited mechanisms explain the spheroidization process simply from the scope of lamellae break-up. This scope of spheroidization is limited since cementite particle coarsening is also involved in soft annealing treatments. Thus, the spheroidization may be defined as a process consisting of two different stages; the first being the mentioned lamellae break-up, and the second, the subsequent dissolution and coarsening of the fragmented cementites, referred to as the Ostwald ripening process [16].

The influence of the initial microstructure on cementite spheroidization has been studied before, but in most of the research works, the microstructures have been obtained by continuous cooling from a given austenitization temperature. On continuous cooling, phase transformation takes place within a range of temperatures that depend on the cooling rate applied. Thus, the microstructural features that are related to the temperature (interlamellar spacing, cementite thickness or length) are not homogeneous, but different as the phase transformation initiates at different times on cooling.

This study analyzes the influence of the initial microstructure obtained by isothermal phase transformation (coarse pearlite, fine pearlite, bainite, and martensite) on cementite spheroidization in a subcritically annealed AISI 5140 steel. As phase transformation is completed at the same temperature, the cementite features are expected to be more homogeneous than when continuously cooled, so that a better comparison between microstructures can be established. The aim of the work is to analyze the effect of the initial microstructure on the cementite spheroidization of an AISI 5140 steel.

\section{Experimental}

The steel studied is a medium carbon chromium hot-rolled steel (AISI 5140). The steel composition is $0.4 \mathrm{C}-0.17 \mathrm{Si}-0.78 \mathrm{Mn}-1.02 \mathrm{Cr}-0.025 \mathrm{~S}$ (in wt \%). Heat treatments were conducted on cylindrical samples $8 \mathrm{~mm}$ in length and $4 \mathrm{~mm}$ in diameter machined along the axis of a hot-rolled $25 \mathrm{~mm}$ diameter rod. The treatments were applied in a dilatometer equipped with an induction heating system. The specimens were austenitized at $850{ }^{\circ} \mathrm{C}$ for $15 \mathrm{~min}$, cooled down at a rate of $20^{\circ} \mathrm{C} / \mathrm{s}$ to a given temperature, and maintained there 
for different times before cooling down to room temperature at $1{ }^{\circ} \mathrm{C} / \mathrm{s}$. The temperature was monitored by a thermocouple fixed on each sample, and the cooling was conducted by pumping He into the chamber. The holding temperatures of 700,630 , and $500{ }^{\circ} \mathrm{C}$ were selected to produce coarse pearlite, fine pearlite, and upper bainite microstructures, respectively. The martensitic microstructure was obtained by direct quenching from $850^{\circ} \mathrm{C}$.

When cooling down from the austenitization temperature, no phase transformation takes place, as shown in Figure 1a. The holding times were selected to assure that 90 pct of the austenite has transformed. In this manner, possible initial spheroidization phenomena related to longer holding times is avoided. Assuming equivalence between the transformed fraction and sample dilatation, the representation in Figure $1 \mathrm{~b}$ is obtained, from which the required times were determined.

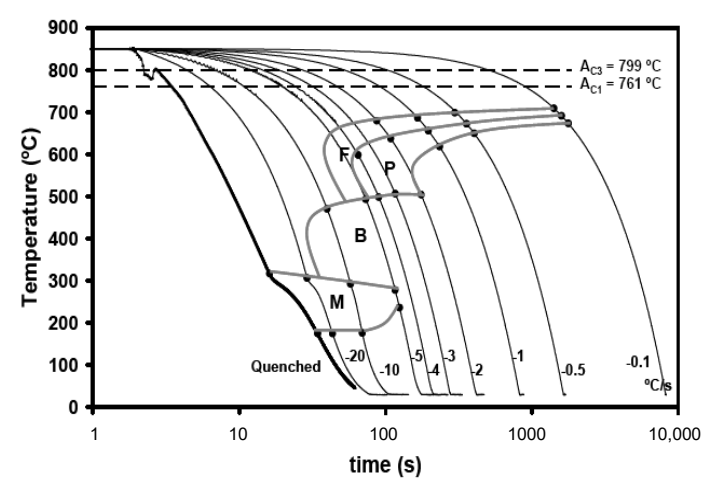

(a)

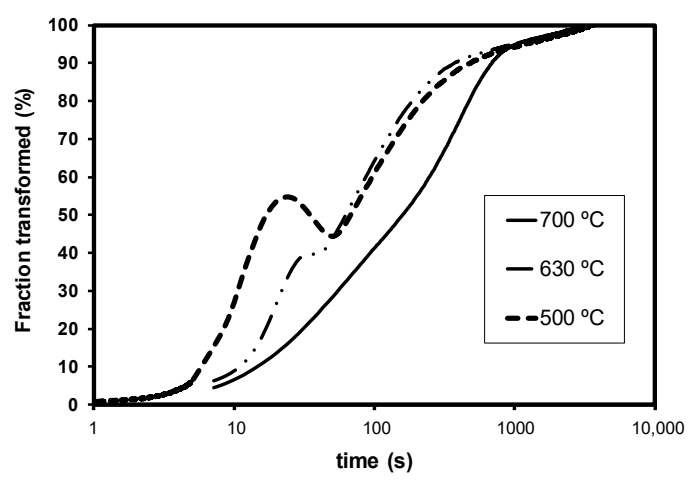

(b)

Figure 1. Continuous cooling diagram (austenitization stage performed at $850{ }^{\circ} \mathrm{C}$ ) (a), and transformed fraction on isothermal holding at different temperatures (b).

The specimens were annealed subcritically for $2 \mathrm{~h}$ (short cycle), $6 \mathrm{~h}$ (intermediate cycle), $10 \mathrm{~h}$ (long cycle), or $50 \mathrm{~h}$ (very long cycle). Preliminary dilatometry tests showed that the critical temperature $\mathrm{Ac}_{1}$ of the steel is around $761^{\circ} \mathrm{C}$. Considering that temperature, and taking into account that in the presence of segregations austenitization may take place at temperatures lower than the expected, an annealing temperature of $720^{\circ} \mathrm{C}$ was selected. The samples were introduced once the temperature in the furnace was stabilized, and they were taken out and air-cooled at room temperature after the specified holding times.

The degree of spheroidization was quantified by image analysis over randomly selected Field emission gun scanning electron microscope (FEG-SEM) micrographs (at 10,000 times magnification) taken from polished samples etched with nital $2 \%$. The image analysis software LEICA QWin provides accurate dimensional data for each cementite detected. As many as necessary micrographs were analyzed in order to measure around $10^{4}$ cementite particles in each condition and obtain an adequate statistical reliability. Particles with an area smaller than $0.001 \mu \mathrm{m}^{2}$ were not considered in the analysis. According to the criteria used by O'Brien and Hosford [22], cementite particles with an aspect ratio lower than 3 were considered to be spheroidized. Thus, the overall degree of spheroidization was determined as the ratio between the area constituted by the spheroidized cementites and the total cementite area (spheroidized and non-spheroidized), normalized to area percentage.

\section{Results}

The coarse pearlite, as shown in Figure 2a, exhibits the classical appearance, i.e., parallel arrangement of the cementite lamellae which constitute the pearlite in each colony. Despite this classic feature, there are some cementites within the colony that exhibit kinked forms. In the fine pearlite, as shown in Figure 2b, microstructure cementite lamellae appear fragmented as they rarely, if ever, cover the whole colony from side to side as occurred in the coarse pearlite. The average interlamellar spacing in the coarse and fine pearlite is $0.27 \mu \mathrm{m}$ 
and $0.10 \mu \mathrm{m}$, respectively. The lamellae thickness is also affected by the transformation temperature, thus, in the coarse pearlite the value is $0.10 \mu \mathrm{m}$, while in the fine pearlite it is $0.06 \mu \mathrm{m}$. The upper bainite features are noticeable in Figure $2 \mathrm{c}$, corresponding to the pre-treatment performed at $500{ }^{\circ} \mathrm{C}$. The ferrite is arranged parallelly, and the carbon rejection from the ferrite has led to the formation of discontinued rows of cementite along the ferrite interfaces. At higher magnification, the presence of small pearlite islands is observed, consisting of very fine and low-spaced cementites, which have nucleated at some prior austenite grain boundaries. However, the fraction of these pearlite islands is low, so the microstructure can be considered as fully bainitic. In Figure $2 \mathrm{~d}$, the martensitic microstructure obtained after imposing a quenching with He from the austenitization temperature of $850^{\circ} \mathrm{C}$ is shown. The lath-like nature of the martensite may be observed where parallel alignment of several laths along the prior austenite grain is noticeable.

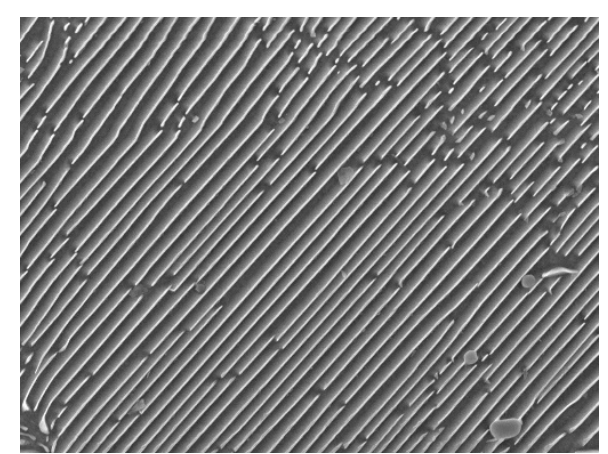

(a)

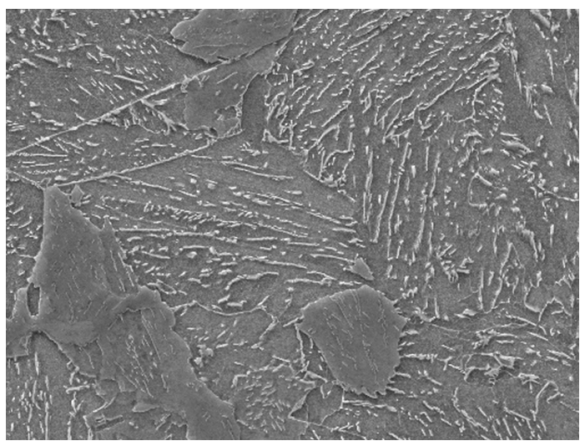

(c)

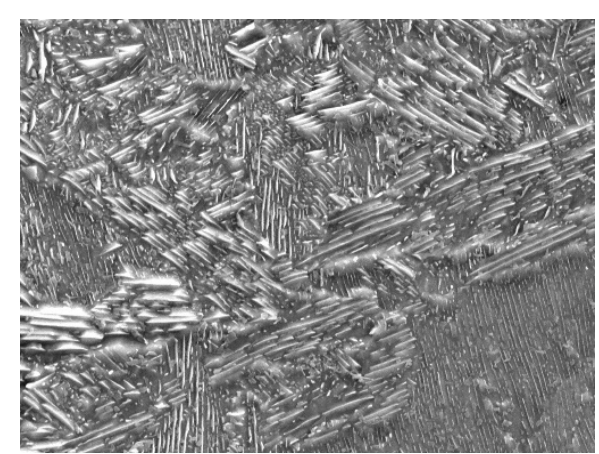

(b)

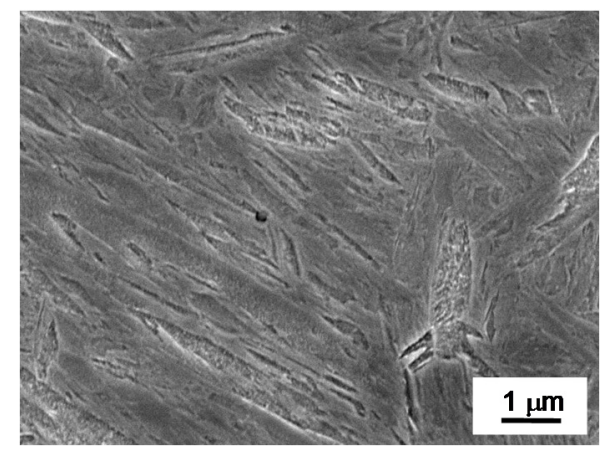

(d)

Figure 2. Microstructures obtained by quick cooling from $850^{\circ} \mathrm{C}$ to the transformation temperature, and subsequent holding at the transformation temperature to promote isothermal phase transformation. Initial coarse pearlite (phase transformation temperature $=700^{\circ} \mathrm{C}$ ) (a), initial fine pearlite (phase transformation temperature $\left.=630^{\circ} \mathrm{C}\right)(\mathrm{b})$, initial bainite (phase transformation temperature $=500{ }^{\circ} \mathrm{C}$ ) (c), and martensite obtained after quenching from $850^{\circ} \mathrm{C}(\mathrm{d})$.

Figure 3 shows the microstructure of the coarse and fine pearlite microstructure after the application of short and long spheroidization cycles. In the coarse pearlite, the exposure to annealing temperatures at $720{ }^{\circ} \mathrm{C}$ produces the lamellae fragmentation, although the microstructure maintains the pearlite features from the beginning; that is, cementite lamellae exhibit straight and elongated morphology, as shown in Figure $3 a$. The lamella break-up proceeds in high curvature areas like kinks that correspond to the energetically more favored positions along the lamella [30]. After the long cycle, the lamellae appear highly fragmented, as shown in Figure 3b. Increasing the treatment maintenance has allowed sufficient time for further lamellae break-up. The microstructure is quite heterogeneous since the spheroidization process progresses at a different rate depending on the colony. Thus, certain pearlite colonies appear highly decomposed, whereas in others a lamellar structure is still present. Even after the application of a very 
long cycle, as shown in Figure 3c, there are cementites that remain which exhibit very high aspect ratios.

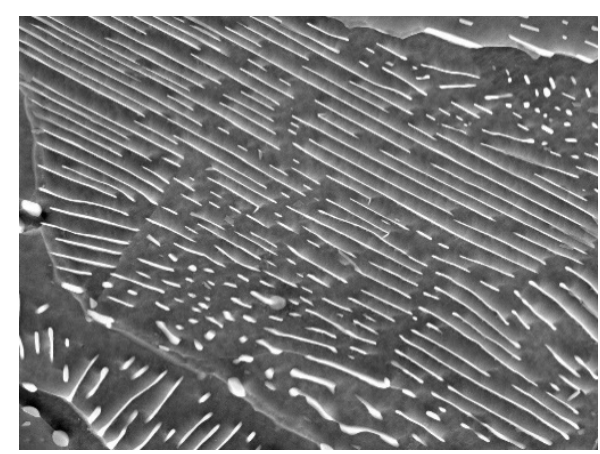

(a)

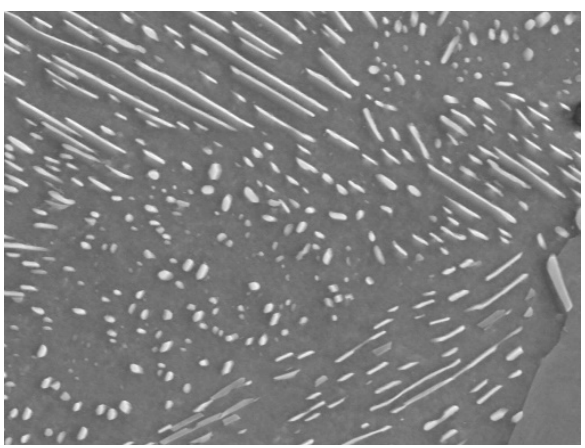

(b)

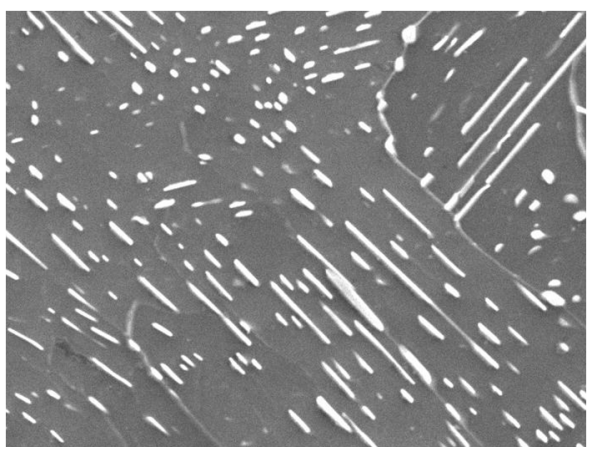

(c)

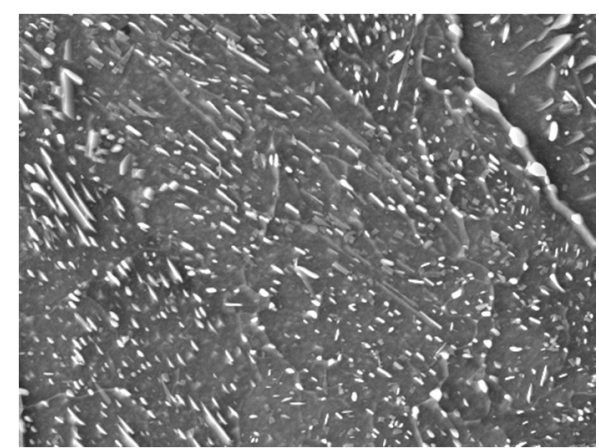

(d)

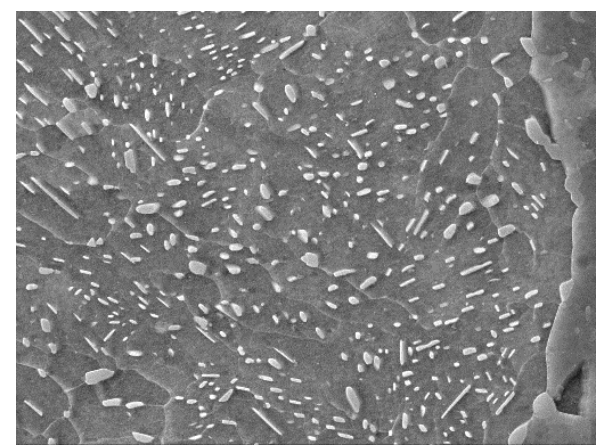

(e)

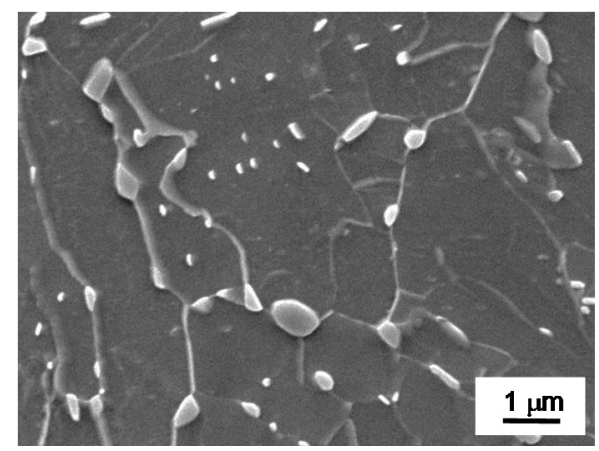

(f)

Figure 3. Coarse pearlite microstructure after short (a), long (b), and very long (c) spheroidization cycle. Idem for fine pearlite in $(\mathbf{d}-\mathbf{f})$.

Unlike in the coarse pearlite, the morphological changes in the fine pearlite are more noteworthy even after the application of the short annealing cycle, as shown in Figure 3d. Despite the shortness of the cycle imposed, the lamellar nature of the cementites has been widely altered into a more globular shape. In spite of that, there are some areas in which straight and long cementites may also be observed. The effective lamellae fragmentation gives rise to the development of high density globular cementite dispersion. After a long spheroidization treatment, an almost fully spheroidized microstructure is obtained in the fine pearlite, as shown in Figure 3e. The presence of lamellar structures is practically negligible; instead, globular and well distributed cementite distribution is achieved. The population constituted by the smallest cementites formed after the initial break-up during the short spheroidization dissolved in the matrix, whereas larger ones located at grain boundaries have coarsened. When the very long annealing cycle is applied, total spheroidization is achieved and cementite coarsening progresses even 
further, especially in the ferrite grain boundaries given the fast carbon diffusion along such boundaries (pipe diffusion), as shown in Figure $3 \mathrm{~d}$.

The bainite microstructure after the application of the short annealing cycle at $720{ }^{\circ} \mathrm{C}$ is shown in Figure 4a. The cementite row-like structure split quickly into sets of individual cementites lineally arranged. Certain small size cementite dissolution and coarsening of those located at the boundaries is appreciated. After the long spheroidization cycle, bainite lath decomposition occurs and cementite coarsening becomes evident at lath boundaries and even within the prior laths, as shown in Figure $4 \mathrm{~b}$. In the case of the martensite microstructure, following the short annealing cycle, very fine cementite and homogeneous distribution is observed, as shown in Figure 4c. The elongated packet-lath morphology persists in the microstructure, and the cementite precipitates mainly at ferrite lath boundaries driven by the carbon depleting from the lath interiors, rather than within the laths. The vast majority of the cementites exhibit globular shapes, although there are also some noticeable cementites that present elongated forms in parallel with the laths. The application of a long annealing treatment results in further cementite coarsening as shown in Figure $4 \mathrm{~d}$. The coarsening is particularly effective at triple points. Prior lath martensite features are almost removed, and not only equiaxed grain formation, but also grain growth are present.

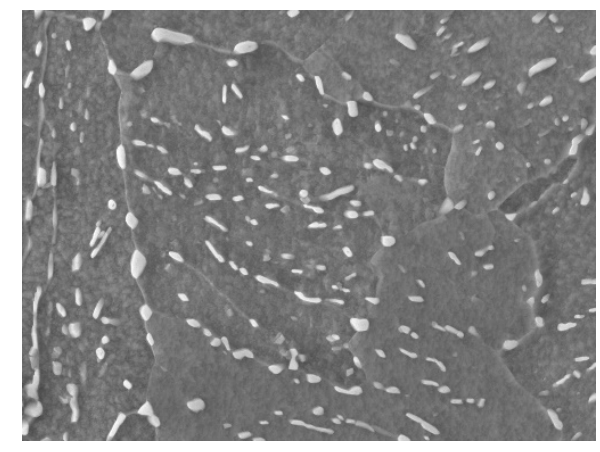

(a)

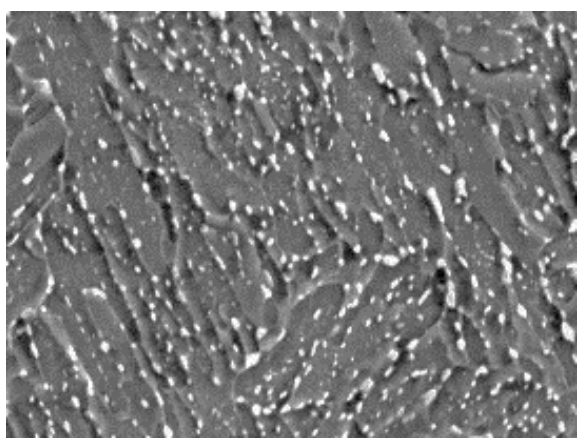

(c)

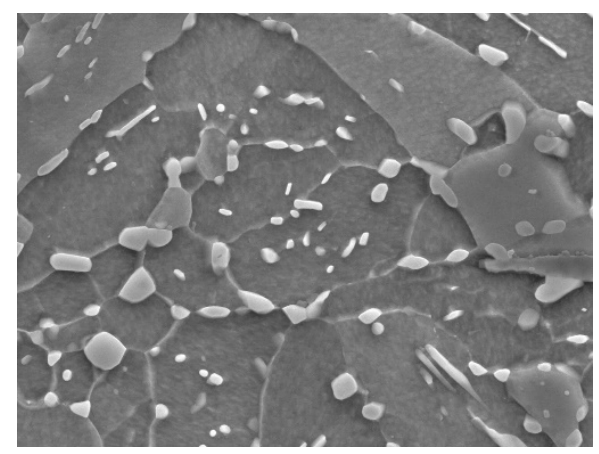

(b)

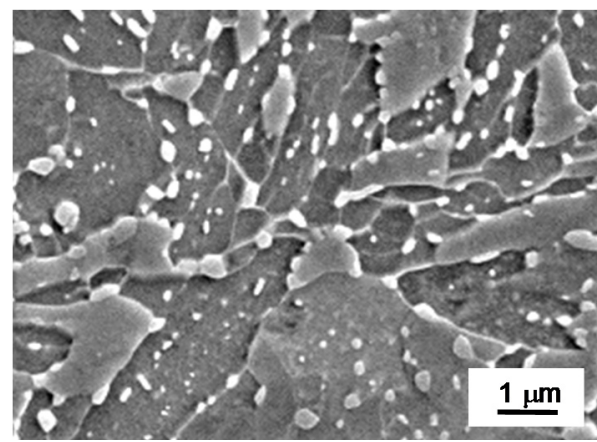

(d)

Figure 4. Bainite microstructure after short (a) and long (b) annealing treatments. Idem for martensite microstructure in $(\mathbf{c}, \mathbf{d})$.

The evolution of the degree of spheroidization for the initial microstructures considered during the different annealing treatments is shown in Figure 5. In Figure 5a, the degree of spheroidization determined as a spheroidized cementite area fraction is represented. As seen, the lower the transformation temperature, the higher the tendency of the microstructure for spheroidization. In the coarse pearlite, the spheroidization degree increases slowly with time, and slows down beyond $6 \mathrm{~h}$. In fine pearlite and bainite microstructures, the spheroidization degree increases quickly in the first hours of treatment, and in both cases similar values are obtained. The degree determined as spheroidized cementite frequency is shown in Figure 5b. Similar trends as in Figure 5a are observed, although the degree obtained by this criterion provides higher values. 


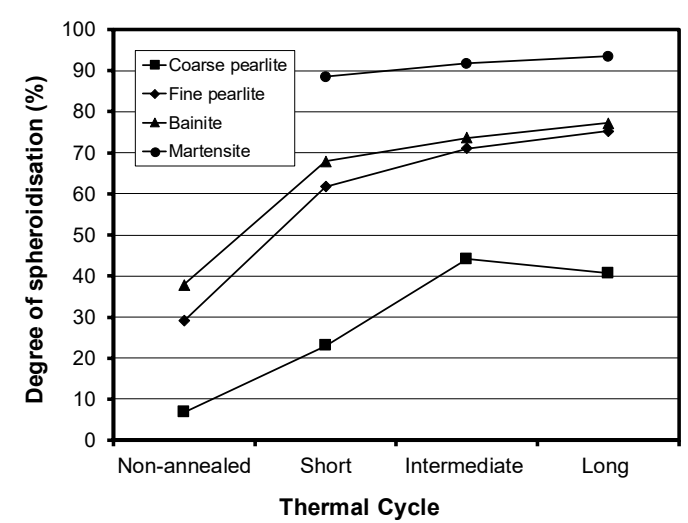

(a)

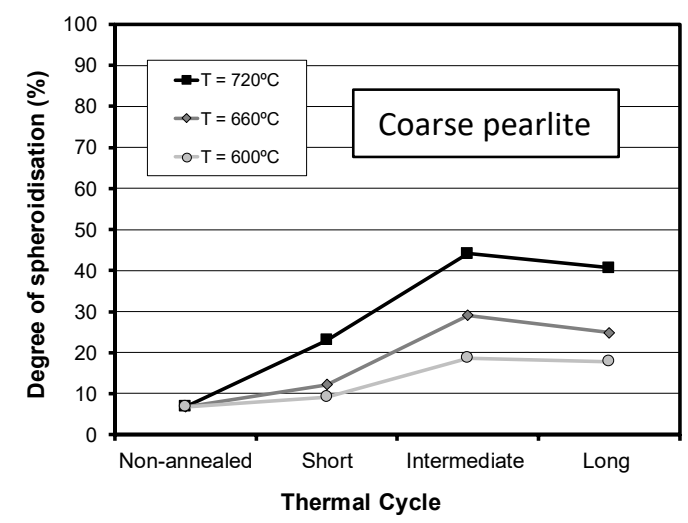

(c)

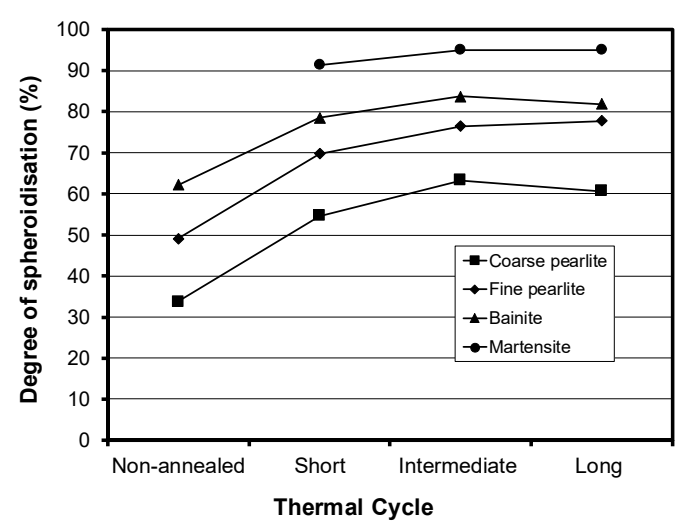

(b)

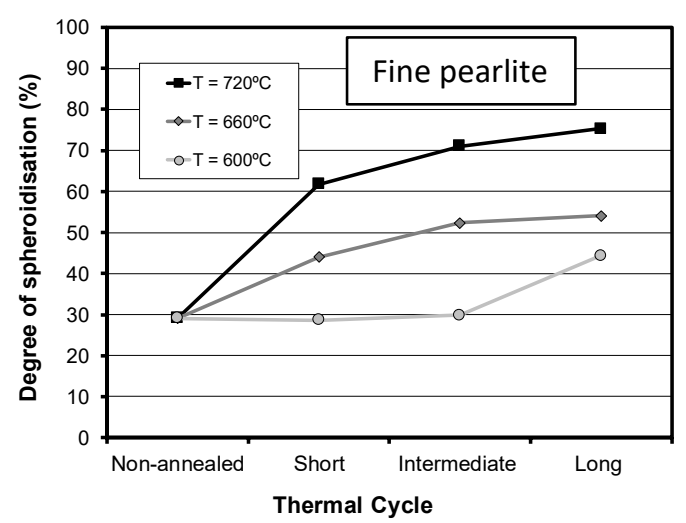

(d)

Figure 5. Evolution of the degree of spheroidization determined as spheroidized cementite area fraction (a) and spheroidized cementite frequency (b) for different initial microstructures. Influence of the annealing temperature on the spheroidization degree determined as spheroidized cementite area fraction for coarse (c) and fine (d) pearlite initial microstructure.

As expected, the spheroidization degree of the microstructures depends strongly on the annealing treatment temperature applied due to diffusion controlled mechanisms involving the spheroidization process. In the case of the initial coarse pearlite, the evolution of spheroidization degree as a function of the treatment temperature is shown in Figure $5 \mathrm{c}$. As seen, the spheroidization degree is drastically reduced with the decrease of the treatment temperature. The same effect is also observed in the case of the initial fine pearlite in Figure 5d.

Microstructure hardness has been evaluated by using a Vickers indenter and an applied load of $1 \mathrm{~kg}$. The hardness values obtained after different annealing treatments for each initial microstructure are shown in Figure 6a. The coarse pearlite shows the lowest values of all the microstructures despite experiencing a low softening on annealing. Such low hardness might be associated, at least partially, with the proeutectoid ferrite content in this microstructure (about 25\%). Fine pearlite instead undergoes a significant drop in hardness after short annealing and a more progressive softening as the annealing cycle duration increases. The bainitic microstructure shows a similar trend to the fine pearlite, and the hardness values are slightly lower than in the former after short and intermediate annealing cycles. Such differences reduce for long and very long annealing, and even the martensite microstructure shows very similar values. For fine pearlite, bainite, and martensite, a long annealing cycle results in a microstructure hardness in the range of 185-195 HV, whereas the hardness for the coarse pearlite is just $165 \mathrm{HV}$. 


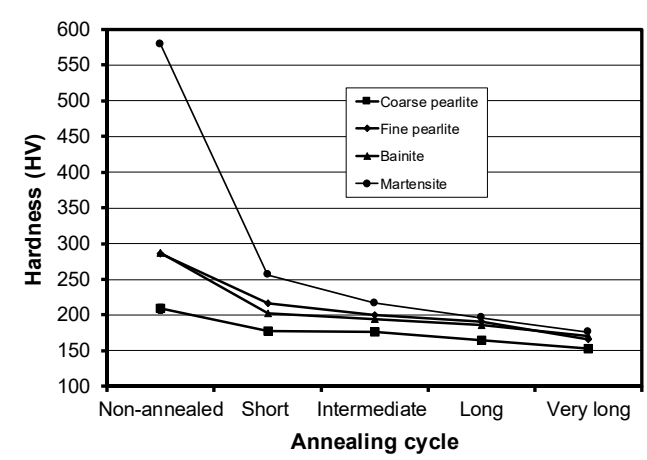

(a)

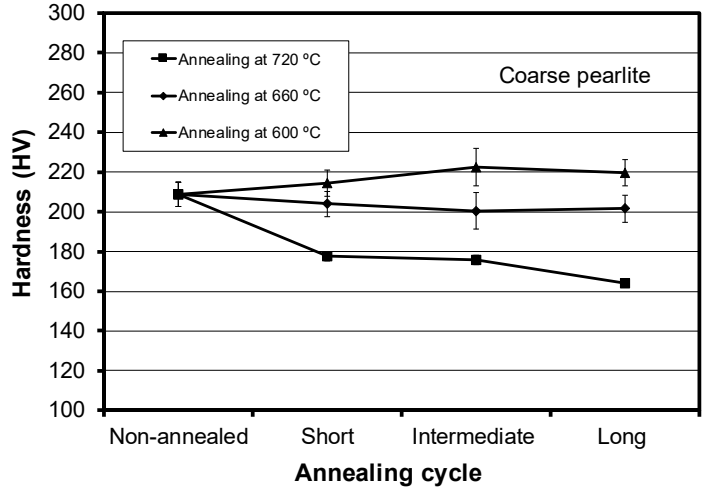

(b)

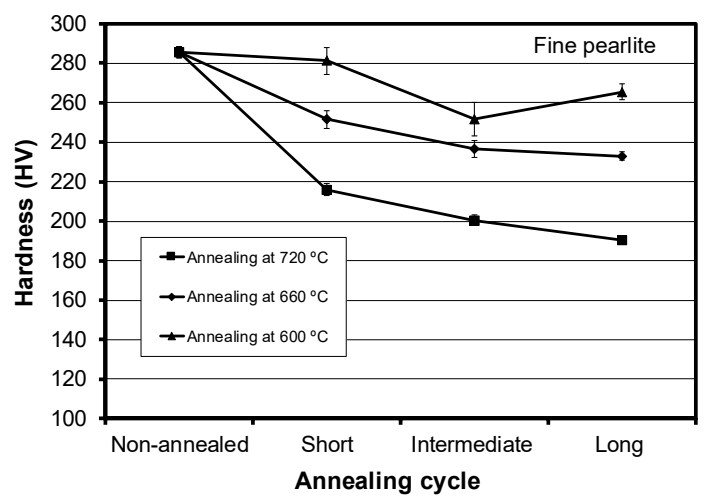

(c)

Figure 6. Hardness evolution after the application of different annealing treatments at $720{ }^{\circ} \mathrm{C}$ depending on the initial microstructure (a), and influence of the treatment temperature on coarse (b) and fine (c) pearlite microstructure.

The influence of the treatment temperature on the microstructure hardness in coarse and fine pearlite is represented in Figure $6 \mathrm{~b}, \mathrm{c}$, respectively. As expected, the softening slows down as the treatment temperature decreases in both cases. In the initial coarse pearlite, the treatments carried out at temperatures below $660^{\circ} \mathrm{C}$ do not produce any softening, and even certain hardening is observed when the treatment is performed at $600{ }^{\circ} \mathrm{C}$. The fine pearlite microstructure is more prone to softening and a significant drop in hardness occurs at both annealing temperatures. Nevertheless, the resulting microstructures exhibit relatively high hardness values ( $>230 \mathrm{HV}$ ) even after applying long and very long annealing cycles at these temperatures.

\section{Discussion}

The presence of defects (such as holes, kinks, and lamella terminations) within the cementite lamella constitutes a starting point for the spheroidization and cementite lamella break-up [24]. In Figure 7a, the presence of holes in the lamellae corresponding to the coarse pearlite can be observed. The cementite plane is quite close to the observation plane and that has allowed for the capture of such holes which otherwise would remain hidden. The curvature along the edge implies a thermal instability of the edge given the different solute concentration in equilibrium in the tip constituted by the edge and the cementite flat area [29]. Thus, a solute transfer is activated from the tip, which causes the dissolution at the tip and the consequent hole growth. Figure $7 \mathrm{~b}$ would constitute an advanced stage of the process described. During growth, the hole may well coalesce with another adjacent hole in the same lamellae, reaching the lamella termination, and causing lamella division in shorter units. 


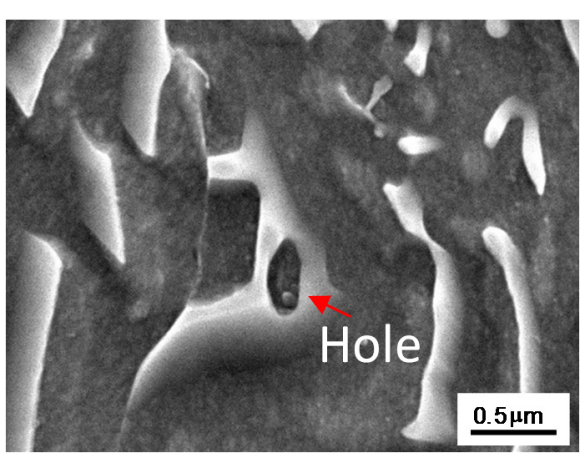

(a)

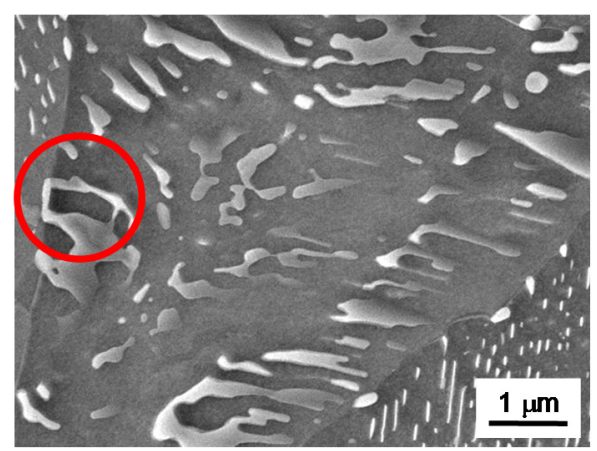

(b)

Figure 7. Micrograph showing the presence of a hole in a cementite lamella in an initial coarse pearlite microstructure (a). Detail of a hole growth (red circle) after the intermediate cycle causing lamellae break-up according to the migration of the edge of the hole in the coarse pearlite after the intermediate cycle $(\mathbf{b})$.

As well as the holes, the kinks present in cementite are the zones where spheroidization phenomena start in the first place. The kinked constitution of the cementite may result from the release of transformation strain and habit plane interfacial strain that has accumulated as the phase transformation proceeds as reported by Zhang et al. [31]. Figure 8a shows the initial coarse pearlite after deep nital etching. Ferrite dissolution in between the lamellae makes it possible to observe the presence of kinks and striations along the cementite. Due to the high curvature in these kinks, their preferential dissolution takes place very quickly on annealing, and thus an elongated cementite can subsequently divide into smaller units. Previous studies attribute the formation of kinks to defects present in the austenite when phase transformation is taking place [32]. As reported, when the pearlite growing front meets with twins or other crystallographic defects, the growing direction may change drastically to adopt an energetically more favorable orientation. From the scope of the spheroidization kinetics, the presence of kinked and distorted pearlite colonies is beneficial, as the lamellae splitting creates new terminations that accelerate further spheroidization. Figure $8 \mathrm{~b}$ shows the mentioned kinked cementites in the coarse pearlite microstructure annealed for $2 \mathrm{~h}$ at $600{ }^{\circ} \mathrm{C}$. As could be intuited, some of the kinks have dissolved, whereas others remain unaltered due to the lowness of the treatment temperature employed, which slows down the dissolution process.

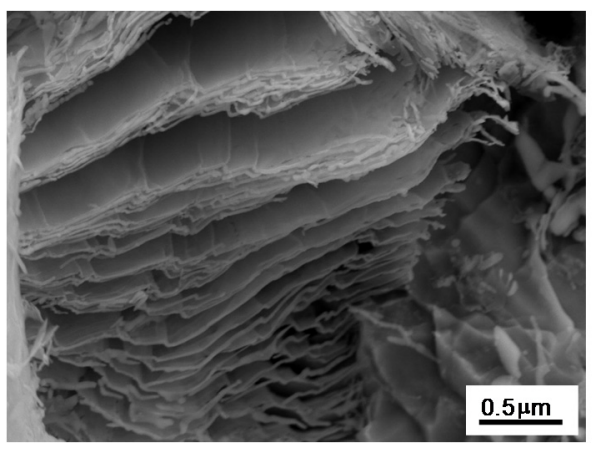

(a)

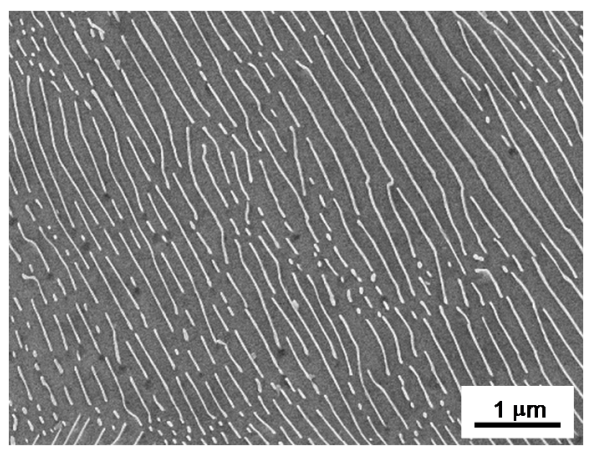

(b)

Figure 8. Presence of kinks along the cementite lamellae (revealed by deep nital etching) (a), and detail of the lamellae break-up initiation due to the kink zone dissolution after the application of a short annealing at $600{ }^{\circ} \mathrm{C}(\mathrm{b})$ in the coarse pearlite.

The lamella terminations act as seeds for the initiation of the spheroidization. Such terminations would arise from the quick dissolution of previous kinks in the lamella or may be due to the sudden interruption of the lamella growth during phase transformation. 
The terminations or the borders of the lamella provide the required curvature for the edge spheroidization, by means of which the edge thickens, driven by the Gibbs-Thomson effect [28]. In Figure 9a, edge spheroidization of the lamellae before break-up is observed. As the flat area of the lamella adjacent to the tip coarsens, a morphological instability is established between the ridge formed along the edge and the remaining flat cementite. As a consequence, the edge splits from the lamella, and the process starts again in the new edge. The break-up would be driven by the reduction of the total $\alpha / \theta$ interface, as described by Rayleigh's instability theory [24]. As a rule of a thumb, the edge spheroidization in both terminations give rise to the formation of two globular shape cementites (one at each side) and a remaining flat cementite that may undergo the same process once and again. When the cementite is sufficiently short after the splitting, no flat cementite could remain. As such, two particular cases could occur depending on the cementite aspect ratio. The first would be represented with an arrow and the letter A in Figure $9 \mathrm{~b}$. The edge spheroidization on both sides of the cementite provokes the lamella to split into two globular cementites. The other case would be that indicated by the letter B. The cementite aspect ratio is lower than in the previous case and the material transfer from the tips to the flat zones result in the formation of a unique globular cementite.

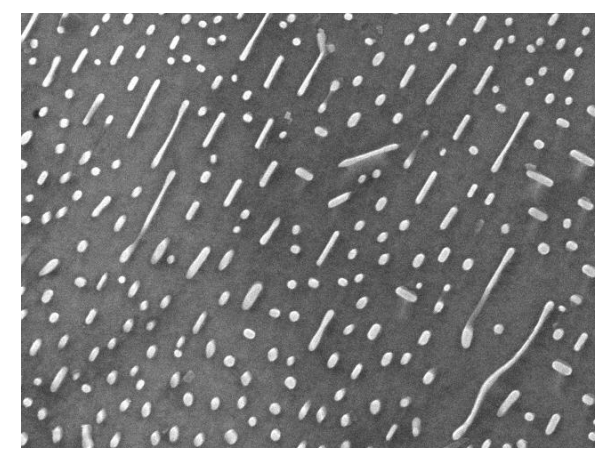

(a)

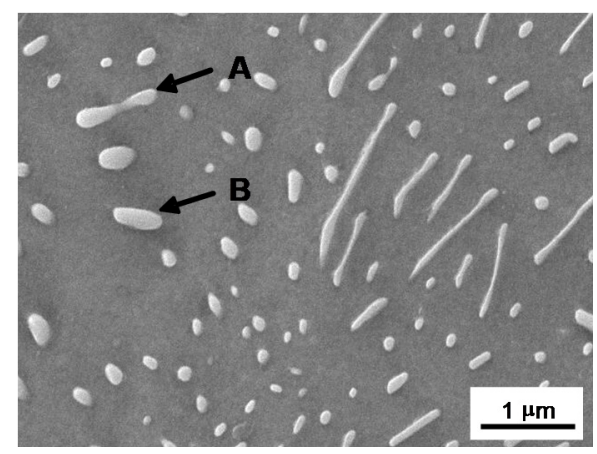

(b)

Figure 9. Cementite edge spheroidization before break-up into globular units (a), and detail of the edge spheroidization phenomenon depending on the cementite lamellae aspect ratio (b), after the intermediate annealing treatment in the coarse pearlite microstructure.

The initial fine pearlite microstructure provides more lamella terminations than the coarse pearlite, where the cementite lamellae are elongated. This difference in termination density could explain the higher and faster spheroidization observed in the fine pearlite, as shown in Figure 5. Furthermore, in the fine pearlite, some of the cementites exhibit relatively low aspect ratios, so they can be converted more quickly into globular shapes. In the coarse pearlite, in contrast, as the cementites are long lamellae, successive edge spheroidization and splitting sequences must take place until the cementite evolves into a set of globular cementites.

Depending on the interlamellar spacing, the solute transfer may well occur between two parallel lamellae as described by the defect migration theory [24] or may simply proceed in a particular lamella. In coarse pearlite, the distance between the cementites after the short cycle spheroidization treatment has not changed considerably with respect to the non-annealed condition (compare Figures $2 a$ and $3 a$ ). The same is observed when the lamella thicknesses are compared. This would imply that there has not been an important material transfer between adjacent lamellae; that is, the solute transfer from the tips to the flat areas takes place exclusively in the same cementite lamella. In contrast, in fine pearlite interlamellar spacing increases and thickening of the elongated cementites could be intuited (compare Figures $2 \mathrm{~b}$ and $3 \mathrm{c}$ ). This would mean that the interlamellar distance is short for interlamellar solute transference.

In the coarse pearlite, the break-up progresses more slowly and long, straight lamellae may remain even after the long annealing cycle, as shown in Figure 3b. In such a case, 
together with the fault migration mechanism, Rayleigh's instability phenomenon gains importance in the spheroidization process in the long term, as high aspect ratio cementite plates persist after the initial stages of the annealing treatment. No evidence of the breakup according to the thermal groove theory has been observed in any of the annealed pearlite microstructures. The process is reported to occur very quickly after subjecting the microstructure to annealing temperatures [27], and consequently the cementite may have broken-up completely before the end of the short annealing treatment.

The initial bainite microstructure consists mostly of elongated cementites joined and aligned in a row along the bainite lath direction. Such particularity provides high curvature areas for rows to split into individual cementite units. Figure 10a shows the presence of such cementite rows in the non-annealed bainite. The break-up proceeds according to the preferential material transference from the necks along the row up to the adjacent zones. Thus, the necks narrow while the neighboring areas enlarge. This situation would correspond to the cementite highlighted by a red circle in Figure 10b, where it can be observed how the cementite thickens at the expense of the continuous dissolution of the neck. This material transfer remains active until the splitting occurs. As a result, almost globular shape cementites are created from an original elongated cementite form.

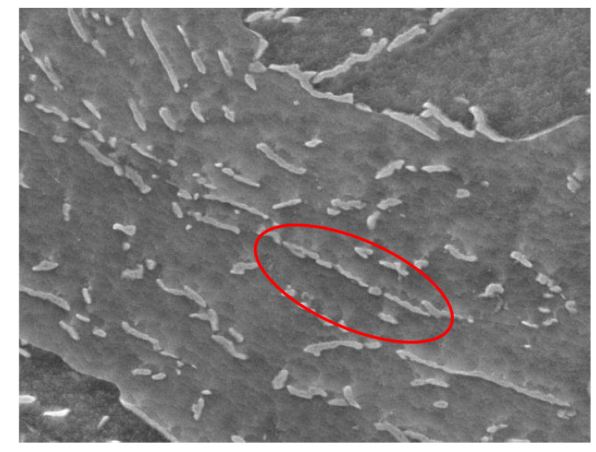

(a)

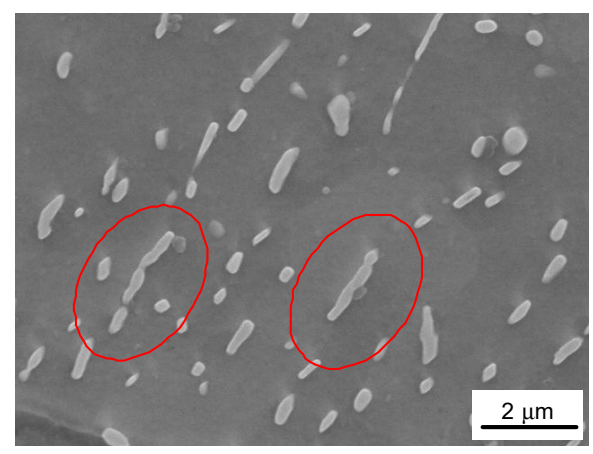

(b)

Figure 10. Cementite spheroidization process in the initial bainite microstructure. Detail of the initial bainite laths in the as-transformed bainite microstructure, where rows of cementites are observed along boundaries between laths as indicated by the red circle (a). Formation of necks during annealing that cause cementite break-up into globular shapes of the cementite rows in the interface between laths (b).

The lattice defects in the matrix due to the higher undercooling condition during bainitic transformation enhance the solute diffusion, leading to a much coarser cementite distribution than in the ferrite-pearlite microstructures for a given annealing cycle, as shown in Figures $3 \mathrm{~b}$,e and $4 \mathrm{~b}$. As for the matrix, it is observed that in some areas where spheroidization has progressed further, recrystallisation occurs, as evidenced by the presence of equiaxed grains. The cementite dispersion throughout the bainite matrix exert a pinning effect that delay the recrystallization taking place in the short term as reported for pearlitic grades subjected to a heavy warm deformation process before annealing [33-38]. As the cementites spheroidize, dissolve, and coarsen during annealing, the energy barrier against recrystallisation diminishes. Cementite dissolution and coarsening allow the dislocation to overcome the obstacles constituted by such cementites, as described by Orowan [39], and recrystallisation could take place.

Considering the ASTM F2282 standard for mechanical fastener manufacturing, the different initial microstructures achieved the spheroidization rating summarized in Table 1. The coarse pearlite microstructure does not achieve the minimum G2/L2 rating that implies an adequate proper workability under any of the annealing cycles studied. In contrast, in either initial fine pearlite or bainite, such a minimum is attained just after the intermediate annealing cycle at $720^{\circ} \mathrm{C}$. In addition, as shown in Figure 6a, these two microstructures after annealing are just about 20-30 HV harder than the softest condition corresponding to 
the coarse pearlite, so the enhanced spheroidization rating is not obtained at the expense of a much harder microstructure. This fact is important from the scope of the tool life employed for cold forming, since higher stresses increase tool wear. Long annealing cycles at $720{ }^{\circ} \mathrm{C}$ give rise to a superior G1 rating in both cases and an additional hardness drop. The initial martensite microstructure achieves the minimum requirement just after the short annealing cycle, and a G0 rating that corresponds to a total spheroidization regarding the standard after a long annealing cycle at $720^{\circ} \mathrm{C}$, after which it softens to values similar to those for fine pearlite and bainite. The application of technology based on quenching after rolling would be impossible in the actual industrial mills and expensive as it involves another step (quenching) in the production. Nevertheless, it is worth emphasizing that the results show that in an industrial conveyor cooling condition, the formation of martensite is not harmful and undesirable whatsoever if the steel wire is subjected to an intermediate or long subcritical annealing treatment.

Table 1. Spheroidization rating of the annealed microstructures according to the ASTM F2282 standard.

\begin{tabular}{|c|c|c|c|c|c|c|c|c|c|c|}
\hline Annealing Temperature $\left({ }^{\circ} \mathrm{C}\right)$ & & & 720 & & & 660 & & & 600 & \\
\hline Annealing Cycle & Short & Inter. & Long & Very Long & Short & Inter. & Long & Short & Inter. & Long \\
\hline Coarse pearlite & $\mathrm{L} 4$ & $\mathrm{~L} 4$ & L3 & L3 & L5 & L5 & $\mathrm{L} 4$ & L5 & L5 & L5 \\
\hline Fine pearlite & G3 & G2 & G1 & G0 & G5 & G4 & G3 & G5 & G5 & G3 \\
\hline Upper bainite & G3 & G2 & G1 & - & - & - & - & - & - & - \\
\hline Martensite & G1 & G1 & G0 & - & - & - & - & - & - & - \\
\hline
\end{tabular}

The cementite spheroidization is a diffusion controlled process, so it is very sensitive to the annealing temperatures and the alloying content. As could be expected from the results in Figure $6 \mathrm{~b}, \mathrm{c}$, the annealing treatments conducted at $660^{\circ} \mathrm{C}$ and $600{ }^{\circ} \mathrm{C}$ do not provide the minimum G2/L2 rating at maintenances shorter than $10 \mathrm{~h}$ in ferrite-pearlite microstructures. The $\mathrm{Cr}$ enriches in the cementite and slows down the diffusion phenomena involving the spheroidization [13].

\section{Conclusions}

- The microstructure evolved at high undercooling exhibits a higher trend for cementite spheroidization. Bainite and fine pearlite microstructures show similar behavior as the applied annealing treatment is prolonged. In both cases, the fastest increase in the degree of spheroidization is achieved after the short annealing treatment. The quenched microstructure allows for the obtaining of practically fully spheroidized microstructures after the application of the annealing treatments. When longer treatments are employed, the spheroidization continues, but it proceeds more slowly. Coarse pearlite exhibits the lowest values, and even after the application of the very long annealing treatment, it maintains the initial pearlite features, that is, long and straight parallel cementite arrangements.

- The initial coarse pearlite does not achieve the minimum G2/L2 spheroidization rating described in the ASTM F2282 standard for the manufacturing of mechanical fasteners involving cold forming operations in any of the annealing treatment conditions studied. Nevertheless, such a minimum rating is relatively quickly obtained, with initial fine pearlite or bainite microstructures treated at $720^{\circ} \mathrm{C}$. This result points towards a different strategy based on the application of faster cooling in industrial procedures to provide a microstructure which is easier to spheroidize.

- The spheroidization in ferrite-pearlite microstructures progresses according to the fault migration theory. Crystallographic defects within the cementite lamellae, such as holes, kinks, or lamella terminations, provide the starting points for the advance of the spheroidization process. In the fine pearlite, the shortness of the cementites gives rise to a higher global cementite termination density than in the coarse pearlite, where such 
imperfections are scarcer, and consequently the formation of spheroidized cementites from an original cementite lamella develops more quickly. The shorter interlamellar distance in the fine pearlite allows for solute transference between the tips to the flat areas of neighboring cementite, resulting in an acceleration of the spheroidization and even coarsening processes. In contrast, in the coarse pearlite, the material transfer is restricted to individual lamella, due to the excessive interlamellar spacing.

- Microstructures soften drastically after the annealing cycle, especially after the short annealing cycle, where the main hardness decrease is observed. The softening in the ferrite-pearlite microstructures derives strictly from the combination of either cementite spheroidization or recovery processes, the latter of which has a more considerable effect in the short term. Despite the fact that the coarse pearlite exhibits the lowest degree of spheroidization, it constitutes by far the softest microstructure of those studied. Bainite and fine pearlite evolve similarly and attain similar hardness values after long annealing treatments.

Author Contributions: Conceptualization, J.A. and J.M.R.-I.; methodology, J.A.; formal analysis, J.A. and J.M.R.-I.; investigation, J.A. and J.M.R.-I.; resources, J.M.R.-I.; writing-original draft preparation, J.A.; writing-review and editing, J.A. and J.M.R.-I. All authors have read and agreed to the published version of the manuscript.

Funding: This research received financial support by the Spanish Science and Innovation Department (CICYT MAT2010-17672 project)

Acknowledgments: Special thanks are given to I. Moran for her help in performing the microhardness tests.

Conflicts of Interest: The authors declare no conflict of interest.

\section{References}

1. ASM Handbook, 14A: Metalworking: Bulk Forming, 10th ed.; ASM International: Materials Park, OH, USA, 2005 ; pp. 383-404.

2. Kwon, O. Static Recrystallization Modelling of Hot Deformed Steels Containing Several Alloying Elements. ISIJ Int. 1992, 32, 350-358. [CrossRef]

3. Ai, J.H.; Zhao, T.C.; Gao, H.J.; Hu, Y.H.; Xie, X.S. Effect of controlled rolling and cooling on the microstructure and mechanical properties of 60Si2MnA spring steel rod. J. Mater. Process. Technol. 2005, 160, 390-395. [CrossRef]

4. Nobari, A.H.; Serajzadeh, S. Modeling of heat transfer during controlled cooling in hot rod rolling of carbon steels. Appl. Therm. Eng. 2011, 31, 487-492. [CrossRef]

5. Lee, Y. Rod and Bar Rolling. Theory and Applications; Marcel Dekker Inc.: New York, NY, USA, 2004.

6. Kazeminezhad, M.; Taheri, A.K. The effect of controlled cooling after hot rolling on the mechanical properties of a commercial high carbon steel wire rod. Mater. Des. 2003, 24, 415-421. [CrossRef]

7. Zhuang, L.I.; Di, W.U.; Wei, L.U. Effects of Rolling and Cooling Conditions on Microstructure and Mechanical Properties of Low Carbon Cold Heading Steel. J. Iron Steel Res. 2012, 19, 64-70.

8. Lee, Y.; Lee, C.; Yoom, E.U. Characteristics of 8T-bolt manufactured by the yield-Ratio-Control-Steel for cold former. Proc. Eng. 2017, 207, 496-501. [CrossRef]

9. Joo, H.S.; Hwang, S.K.; Baek, H.M.; Im, Y.T.; Son, I.H.; Bae, C.M. Manufacturing of medium carbon steel wires with improved spheroidization by non-circular drawing sequence. Proc. Eng. 2014, 81, 682-687. [CrossRef]

10. ASTM International F2282-03. Standard Specification for Quality Assurance Requirements for Carbon and Alloy Steel Wire, Rods, and Bars for Mechanical Fasteners; ASTM International: West Conshohocken, PA, USA, 2009; pp. 4-8.

11. Qian, D.; Yang, J.; Mao, H.; Hua, L. Experiment study on warm ring rolling of 52100 bearing steel coupling microstructure spheroidisation. Proc. Eng. 2017, 207, 1224-1229. [CrossRef]

12. Lü, Z.; Zhang, H.; Qing, M.; Wang, Z.; Fu, W. Effect of Cyclic Annealing on Microstructure and Mechanical Properties of Medium Carbon Steel. J. Iron Steel Res. 2016, 23, 145-150.

13. Szala, M.; Winiarski, G.; Wójcik, L.; Bulzak, T. Effect of Annealing Time and Temperature Parameters on the Microstructure, Hardness, and Strain-Hardening Coefficients of 42CrMo4 Steel. Materials 2020, 13, 2022. [CrossRef]

14. Mondal, D.K.; Dey, R.M. Effect of structures on the response of spheroidization in a eutectoid plain carbon steel. Trans. Ind. Inst. Met. 1984, 37, 351-356.

15. Bhadeshia, H.K.D.H. Steels for bearings. Prog. Mater. Sci. 2012, 57, 268-435. [CrossRef]

16. Krauss, G. Steel: Processing, Structure, and Performance; ASM International: Materials Park, OH, USA, 2005.

17. Nasiri, Z.; Mirzadeh, H. Spheroidization heat treatment and intercritical annealing of low carbon steel. J. Min. Metall. Sect. B Metall. 2019, 55, 405-411. [CrossRef] 
18. Hernández-Silva, D.; Morales, R.D.; Cabañas-Moreno, J.G. The Spheroidization of Cementite in a Medium Carbon Steel by Means of Subcritical and Intercritical Annealing. ISIJ Int. 1992, 32, 1297-1305. [CrossRef]

19. Chattopadhyay, S.; Sellars, C.M. Kinetics of pearlite spheroidisation during static annealing and during hot deformation. Acta Metall. 1982, 30, 157-170. [CrossRef]

20. Karadeniz, E. Influence of different initial microstructure on the process of spheroidization in cold forging. Mater. Des. 2008, 29, 251-256. [CrossRef]

21. Hwang, H.; De Cooman, B.C. Influence of the Initial Microstructure on the Spheroidization of SAE 52100 Bearing Steel. Steel Res. Int. 2016, 87, 112-125. [CrossRef]

22. Chou, C.C.; Kao, P.W. Improvement of Mechanical Properties of Spheroidized 1045 Steel by Induction Heat Treatment. J. Mater. Sci. 1986, 21, 3339-3344. [CrossRef]

23. Parusov, V.V.; Dolzhenkov, I.I.; Evsyukov, M.F. Investigation of transformation kinetics of supercooled austenite into globular pearlite. Russ. Metall. 1978, 3, 102-108.

24. O'Brien, J.M.; Hosford, W.F. Spheroidization cycles for medium carbon steels. Met. Trans. A 2002, 33, 1255-1261. [CrossRef]

25. Nichols, F.A.; Mullins, W.W. Surface-(interface-) and volume-diffusion contributions to morphological changes driven by capillarity. Trans. AIME 1965, 233, 1840-1848.

26. Amos, P.G.K.; Battacharya, A.; Nestler, B.; Ankit, K. Mechanisms of pearlite spheroidization: Insights from 3D phase-field simulations. Acta Mater. 2018, 161, 400-411. [CrossRef]

27. Tian, Y.L.; Kraft, R.W. Mechanisms of Pearlite Spheroidization. Met. Trans. 1987, 18, 1403-1414. [CrossRef]

28. Wang, Y.T.; Adachi, Y.; Nakajima, K.; Sugimoto, Y. Quantitative three-dimensional characterization of pearlite spheroidization. Acta Mater. 2010, 58, 4849-4858. [CrossRef]

29. Malzahn Kampe, J.C.; Courtney, T.H.; Leng, Y. Shape instabilities of plate-like structures-I. Experimental observations in heavily cold worked in situ composites. Acta Metall. 1989, 37, 1735-1745. [CrossRef]

30. Sharma, G.; Ramanujan, R.V.; Tiwari, G.P. Instability mechanisms in lamellar microstructures. Acta Mater. 2000, 48, 875-889. [CrossRef]

31. Zhang, M.-X.; Kelly, P.M. Crystallography and morphology of Widmanstätten cementite in austenite. Acta Mater. 1998, 46, 4617-4628. [CrossRef]

32. Hackney, S.A.; Shiflet, G.J. Pearlite growth mechanism. Acta Metall. 1987, 35, 1019-1028. [CrossRef]

33. Arruabarrena, J.; López, B.; Rodriguez-Ibabe, J.M. Influence of Prior Warm Deformation on Cementite Spheroidization Process in aLow-Alloy Medium Carbon Steel. Met Trans. 2014, 45, 1470-1484. [CrossRef]

34. Arruabarrena, J.; López, B.; Rodriguez-Ibabe, J.M. Influence of DIT Parameters on the Cementite Spheroidization in a Low-Alloy Medium Carbon Steel. Met. Trans. 2016, 47, 412-423. [CrossRef]

35. Tsuji, N.; Maki, T. Enhanced structural refinement by combining phase transformation and plastic deformation in steels. Scr. Mater. 2009, 60, 1044-1049. [CrossRef]

36. Handa, K.; Kimura, Y.; Yasumoto, Y.; Kamioka, T.; Mishima, Y. Effect of Deformation and Annealing Temperatures on Ultrafine Microstructure Development and Yield Strength of Pearlitic Steel Through Continuous Recrystallization. Mater. Sci. Eng. A 2010, 527, 1926-1932. [CrossRef]

37. Song, R.; Ponge, D.; Raabe, D.; Kaspar, R. Microstructure and crystallographic texture of an ultrafine grained C-Mn steel and their evolution during warm deformation and annealing. Acta Mater. 2005, 53, 845-858. [CrossRef]

38. Storojeva, L.; Kaspar, R.; Ponge, D. Effects of Heavy Warm Deformation on Microstructure and Mechanical Properties of a Medium Carbon Ferritic-Pearlitic Steel. ISIJ Int. 2004, 44, 1211-1216. [CrossRef]

39. Humphreys, F.J.; Hatherly, M. Recrystallization and Related Annealing Phenomena, 2nd ed.; Elsevier: Oxford, UK, 2004. 